\section{Cardiovascular CT and MR Imaging}

\author{
C. Catalano, M. Anzidei, and A. Napoli, Eds.
}

New York, NY: Springer, 2013, 362 pages, \$239

The basic purpose of Cardiovascular CT and MR Imaging is to provide practical information on how to apply CT angiography (CTA) and MR angiography (MRA) in daily clinical practice. In the application of these techniques, correct methods of image acquisition for each target organ are of the utmost importance. Thus, this book focuses on acquisition mechanisms and protocols, including the use of contrast material and the reconstruction of images. Another purpose of the book is to enable clinicians to understand the results of CTA and MRA. For this purpose, the basic pathophysiology of vascular diseases and their image findings are also covered.

The main target audience of the book is physicians in the field of cardiovascular radiology who want to understand and use CTA and MRA in clinical practice. Currently, the use of these two technologies is inevitable in many vascular diseases, and physicians should have an understanding of how they work, when they are indicated, and how the images should be interpreted. The book can also provide essential information for residents in cardiology, radiology, and nuclear medicine, as well as for students and technologists in the fields of medicine and medical imaging. It also would be a perfect handbook for experienced cardiologists, radiologists, and radiologic technologists.

The first part of the book discusses the principles of CTA and MRA, including the use of contrast material, MR sequences, and postprocessing. Although not thoroughly discussed in detail, imaging physics and algorithms are explained enough to give nonradiologist clinicians and residents a basic understanding. A reader will at least be able to communicate with radiologists or technologists in the field of vascular radiology after finishing this book.

The remainder of the book is on the clinical application of CTA and MRA. Each chapter discusses the imaging of particular anatomic sites, such as the intracranial circulation, thoracic aorta, coronary circulation, and upper-limb arteries. Some chapters discuss specific diseases such as vascular malformations and vasculitis. Techniques are explained in terms of patient preparation and image acquisition protocols. The discussion of applications in specific diseases covers the basic facts of the disease, the clinical picture and diagnosis, imaging and reporting, and treatment. Thus, a reader can understand how to obtain, interpret, and report images of most vascular disorders covered in the book, which include atherosclerosis and plaque, vascular obstruction of various causes, aneurysm, malformations, rupture, dissection, vasculitis, trauma, surgical interventions, and cardiac valvular disorders.
A pragmatic explanation is given of the technology of CTA and MRA. The discussion of the theoretic background and algorithms for image acquisition and reconstruction is not in great depth but is sufficient. Although 3-dimensional volume renderings and curved planar reformations of the images are, at a glance, beautiful, a basic understanding of image technology is required to correctly interpret these images. However, readers who are not experts in imaging physics may become lost in a too-detailed discussion of technology. The authors of this book do not go too far in either direction. Instead, they focus on patient preparation, imaging settings and sequences, and other topics that actually are required for practical applications. Details on imaging technology and protocols are covered not only in the first part of the book but in each of the remaining chapters.

A highlight of the book is that each of the clinical application chapters is formatted using the same structure, presenting first anatomy, then technique, and finally specific diseases in terms of clinical aspects, image findings, and treatment. This consistency in structure is helpful to readers who wish to use the book as a handbook and will be beneficial in helping beginners comprehensively understand the application of CTA and MRA to specific diseases.

Another highlight of the book is the many figures it includes-more than 400, most of which are of CTA and MRA cases. As a result, the book could be regarded almost as a kind of atlas on cardiovascular diseases. Most of the case images are of high quality and are excellent examples of the explained cases. The authors' endeavor to collect optimal example cases is notable. Additionally, there are many colorful schematic drawings that are beautiful and well drawn and many tables that summarize image parameters, disease classifications, and other categories. With all these figures and tables, the book makes it easy to systematically understand vascular diseases and their image findings.

This book may not be sufficient for specialized radiologists and cardiologists who are experienced in vascular disorders. There is no thorough review of the literature or in-depth discussion on the role of CTA and MRA in vascular disorders. However, although the book is only about 350 pages, it contains much basic essential information on the use of these techniques in vascular disorders. Cardiovascular CT and MR Imaging can serve as an ideal guide for beginners or as a handbook for experts.

\section{Jin Chul Paeng}

Seoul National University Hospital

101 Daehak-ro, Jongno-gu

Seoul, 110-744, Korea

E-mail: paengjc@snu.ac.kr

Published online Aug. 22, 2013.

DOI: 10.2967/jnumed.113.129296 